\title{
Computation of Mean Velocity in Open Channels Using Acoustic Velocity Meters
}

By Eduardo Patino and Darwin Ockerman

U.S. GEOLOGICAL SURVEY

Open-File Report 97-220

Tallahassee, Florida

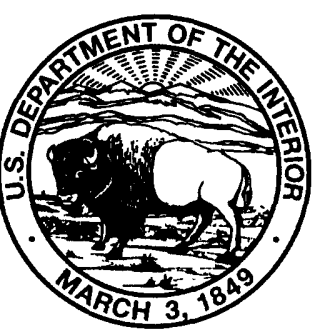




\title{
U.S. DEPARTMENT OF THE INTERIOR BRUCE BABBITT, Secretary
}

\author{
U.S. GEOLOGICAL SURVEY \\ Gordon P. Eaton, Director
}

The use of firm, trade, and brand names in this report is for identification purposes only and does not constitute endorsement by the U.S. Geological Survey.

For addtional information write to:

\section{District Chief}

U.S. Geological Survey

227 North Bronough Street, Suite 3015

Tallahassee, FL 32301
Copies of this report can be purchased from:

U.S. Geological Survey

Branch of Information Services

Box 25286, MS 517

Denver, CO 80225-0046 


\section{CONTENTS}

Abstract
Introduction
Calculating Acoustic Line Velocity
Channel Characteristics and Flow Conditions
Computation of Mean Channel Velocity
Example 1
Example 2
Conclusions

\section{FIGURES}

1. Schematic diagram of acoustic velocity meter transducer positioning along stream banks ................................... 2

2. Typical channel section for acoustic velocity meter installation. ..................................................................

3. Sketch showing effect of changing stream stage on location of acoustic path in relation to vertical velocity profile

4. Sketch showing effect of vertical velocity shapes on the relation of acoustic line velocity to mean channel velocity.

5. Schematic profiles showing vertical and horizontal ray-bending effects on acoustic path length

6. Plan view showing variable flow angles through an acoustic velocity meter gaging site

7. Distorted vertical velocity profile due to tidal effect

8. Multipath applications showing cross-path setup and the horizontal flow angle problem and multiple vertical paths and the velocity profile problem.

9. Plan view showing flow angle caused by vegetation at acoustic velocity meter section and cross-section view showing effective flow area significantly reduced by vegetation at acoustic velocity meter section

10. Graph showing relation of acoustic line velocity to mean channel velocity at site A.

11. Graph showing relation of acoustic line velocity to mean channel velocity for three different stage levels at site B

12. Graph showing relation of velocity ratio $\left(V_{m} / V_{l}\right)$ to stage at site B.

\section{TABLES}

1. Acoustic velocity meter system data and results of regression analysis at site A

2. Acoustic velocity meter system data and results of regression analysis at site $B$ 
$A=$ Flow cross-sectional area;

$B=$ Acoustic path length;

$c=$ Speed of sound in water;

$C=$ The intercept or constant;

$d=$ Depth of acoustic path;

$D_{c}=$ Clearance distance;

$f=$ Acoustic signal frequency;

$H=$ Stage;

$L=$ Acoustic path length;

$L_{I}=$ Direct path length;

$L_{2}=$ Path length with ray bending;

$\Theta=$ Angle of acoustic path with respect to flow;

$P_{n}=$ Other significant variables;

$Q=$ Discharge;

$t_{a b}=$ Acoustic signal traveltime from point $a$ to $b$;

$t_{b a}=$ Acoustic signal traveltime from point $b$ to $a$;

$V=$ Mean velocity of water for the section;

$\bar{V}=$ Mean channel velocity;

$V_{l}=$ Acoustic line velocity;

$V_{m} / V_{l}=$ Velocity ratio;

$X_{i}, X_{n}=$ Regression coefficients;

$W=$ Total channel width; and

$\lambda=$ Wave length. 


\title{
Computation of Mean Velocity in Open Channels Using Acoustic Velocity Meters
}

\author{
By Eduardo Patino and Darwin Ockerman
}

\section{ABSTRACT}

Commercially available acoustic velocity meter (AVM) systems are widely used for discharge computations in streams. Although AVM systems are often viewed as discharge meters, they are actually velocity measuring devices. The accuracy of the measurements depends on the calibration process that relates acoustic line velocity to mean channel velocity. The relation of acoustic line velocity (integrated measurement of velocity along a path between the transducers) to mean channel velocity depends on a number of physical factors (channel characteristics and flow conditions) and is determined from field measurements. Field discharge measurements were made at two AVM sites. Results were used to demonstrate how single and multiple linear regression models can be used to calibrate acoustic line velocity to mean channel velocity and discharge.

\section{INTRODUCTION}

Acoustic velocity meter (AVM) systems have proven to be accurate and reliable instruments for measuring velocities of water in open channels (Laenen and Curtis, 1989). The accuracy of the measurements depends on the calibration process that relates acoustic line velocity to mean channel velocity. AVM systems, often viewed as discharge meters, are actually velocity measuring devices. Discharge in a channel is determined by:

$$
Q=V A
$$

where $Q$ is discharge, $V$ is the mean velocity of water for the section, and $A$ is the flow cross-sectional area.

AVM systems provide a line velocity measurement at one or several points in the vertical. The acoustic line velocity is an integrated velocity measurement along a line or path between the acoustic transducers. This line velocity is usually a reliable index of the actual mean channel velocity used to calculate discharge. Such relations can be complex and can be affected by stage or other factors that change the relative position of the line velocity with respect to the vertical velocity distribution in the channel (Patino, 1996). To accurately relate acoustic line velocity to mean channel velocity and discharge for the flow section, a series of calibration measurements is needed, covering the wide range of flow conditions normally encountered at each site. In addition to the calibrated acoustic line velocity, the stage-area relation for the channel cross section is also necessary to determine discharge.

This report documents a technique for using acoustic line velocity to compute mean channel velocity and water discharge by means of field measurements and regression analyses. Factors that can affect this relation are described along with a model to account for these factors when computing mean channel velocity from acoustic line velocity. Two field examples are presented, demonstrating the use of regression analyses to develop the AVM rating.

\section{Calculating Acoustic Line Velocity}

AVM systems measure the velocity of flowing water by means of an acoustic signal that travels faster downstream than upstream. Traveltimes are measured from point $a$ to $b$ and from point $b$ to $a$ as shown in figure 1 . The difference between these traveltimes is then used in conjunction with the path length (distance from 


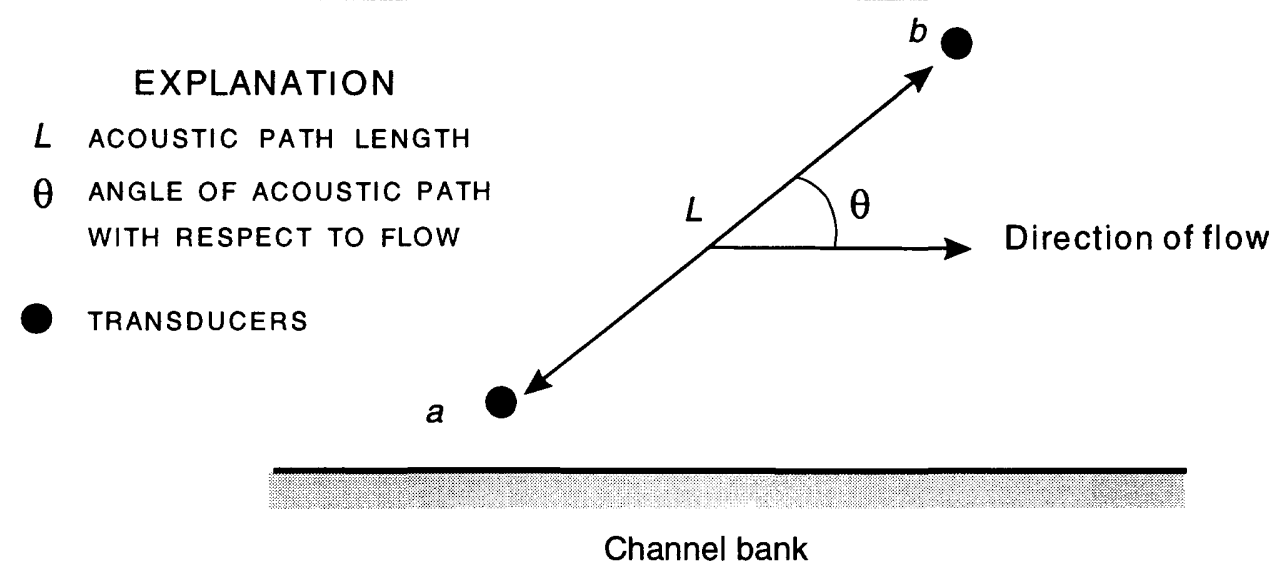

Figure 1. Acoustic velocity meter transducer positioning along stream banks.

one transducer to the other) and the path angle with respect to the flow (measured or estimated) to calculate the velocity component of the acoustic signal in the direction of flow. The general equation for calculating acoustic line velocity is defined by Laenen and Smith (1983) as follows:

$$
V_{l}=\left(\frac{L}{2 \cos \Theta}\right)\left(\frac{1}{t_{b a}}-\frac{1}{t_{a b}}\right)
$$

where $V_{l}$ is the acoustic line velocity at the elevation of the acoustic path, $L$ is the acoustic path length, $\Theta$ is the angle of the acoustic path with respect to flow, $t_{b a}$ is the acoustic signal traveltime from point $b$ to $a$, and $t_{a b}$ is the acoustic signal traveltime from point $a$ to $b$. The velocity, $V_{l}$, measured by the AVM represents an averaged value across the channel or stream from transducer to transducer at a fixed elevation.

\section{Channel Characteristics and Flow Conditions}

Various channel characteristics, flow conditions, and other physical factors can affect the relation of acoustic line velocity to mean channel velocity, and in turn, affect the determination of discharge. The ideal AVM gaging site minimizes most of these factors. The fewer complicating factors involved, the simpler, more reliable, and precise the rating becomes. Physical factors that can affect the relation of acoustic line velocity to mean channel velocity are channel cross-section geometry, stage and acoustic path depth, vertical velocity profiles, temperature and density gradients, unstable flow patterns, and vegetation. These physical factors are described in detail below. Additionally, alternative solutions are provided, where possible, to correct or account for any problems.

Channel Cross-Section Geometry-AVM acoustic paths require a minimum distance below the water surface and above the channel bottom to operate properly; otherwise, signal reflections can occur from the water surface or channel bottom, causing erroneous velocity readings. For wide and shallow streams and channels, the acoustic transducers are often located a distance from shore to reach the depth necessary to avoid surface and bottom interference. Sometimes, a significant part of the channel section cannot be measured by the AVM systems as shown in figure 2. This can greatly influence the relation of acoustic line velocity to mean channel velocity. Even so, acoustic line velocity can still be used as an index for mean channel velocity, as long as discharge measurements, which include the entire flow area, are made during all or most of the hydraulic conditions possible for each specific site and assuming proportionality between the unmeasured and measured parts of the flow section. For gaging sites that have an unstable channel bottom or icecover formation, careful consideration must be given to changes in the cross-sectional area that could affect the acoustic line velocity to mean channel velocity relation.

Stage and Acoustic Path Depth-Water-surface elevation (stage) is a major factor in the proper calibration of AVM systems for two main reasons: (1) minimum clearance requirements between acoustic path and water surface or channel bottom, and (2) position 


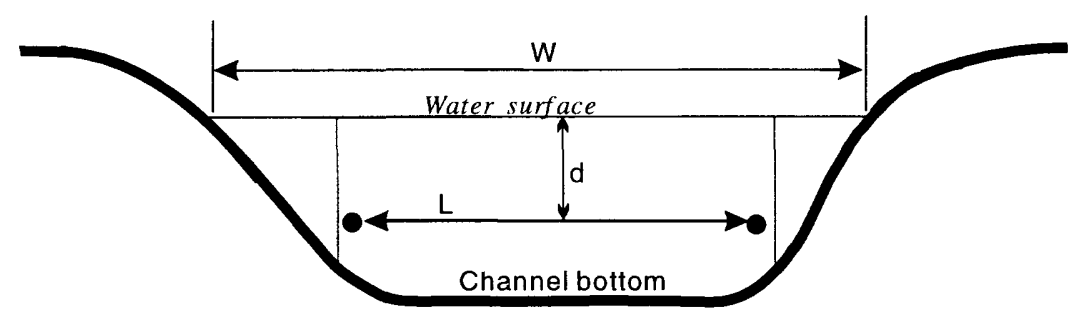

EXPLANATION

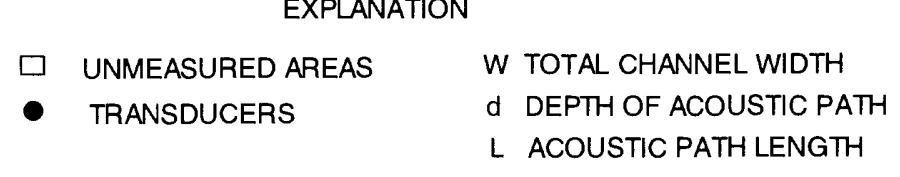

Figure 2. Typical channel section for acoustic velocity meter installation.

of the acoustic path in the vertical velocity profile. The position of the acoustic path can affect the relation of acoustic line velocity to mean channel velocity. Scour and fill or ice-cover buildup can change the relative position of the acoustic path with respect to channel depth, and necessary adjustments are required to account for this effect. During low-stage conditions, proper operation of AVM systems depends on the depth of the transducers and length of the acoustic path. Shorter acoustic paths have less probability of being affected by signal reflections from the water surface or channel bottom, reducing the chances of multipath interference on the acoustic signal. Multipath interfer- ence is defined as the deterioration of the main signal due to reflections at the boundaries. Minimum clearance is calculated as described by Laenen (1985):

$$
D_{c} \cong \sqrt{(\lambda B) / 2}
$$

where $D_{c}$ is clearance distance, $\lambda$ is wave length, and $B$ is acoustic path length. Wave length is computed by $(c / f)$, where $c$ is the speed of sound in water and $f$ is the acoustic signal frequency.

Vertical Velocity Profiles-Vertical velocity profiles depend mainly on channel width, depth, and velocity, but in shallow streams with low velocities, wind or air surface friction can also play an important role in shaping the vertical velocity profile. Ice cover can affect the velocity profile as a result of reduced flow depths and increased friction caused by the ice surface. Scour and fill can also affect the vertical velocity profile. These parameters need to be analyzed to determine their effect on the shape and stability of vertical velocity profiles and to obtain a better calibration of AVM systems. Changes in stage, magnitude of velocity, flow direction, or windspeed and direction can have a direct influence on the shape of vertical velocity profiles, and therefore, on the relation of acoustic line velocity to mean channel velocity. This relation is based on the vertical location of the transducer and the shape of the vertical velocity profile. Three vertical velocity profiles and the vertical location of the acoustic path for high and low stages are shown in figure 3 . The vertical location of the acoustic path in relation to the vertical velocity profile becomes higher with decreasing stage and becomes lower with increasing stage. The acoustic path location in the vertical velocity profile can greatly affect the acoustic line velocity to mean channel velocity relation, depending on the actual vertical velocity distribution at the site.

The magnitude of channel velocity and windspeed and direction can have a strong influence on the relation of acoustic line velocity to mean channel velocity by modifying the shape of the vertical velocity profile, even with no significant change in stage (fig. 4). Discharge measurements at the gaging site need to be made in an attempt to cover the range of hydraulic conditions possible to define the significance of all influencing parameters.

Temperature and Density Gradients-Temperature and density gradients can change the path of the acoustic signal by refraction. This phenomenon, called "ray bending," can affect the acoustic signal in the horizontal or vertical directions (fig. 5). Because the AVM instrument is programmed to compute velocity based on the actual straight-line distance between the transducers, longer refracted paths introduce error in the AVM velocity calculation. 


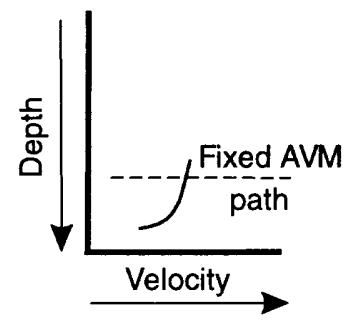

Low Stage

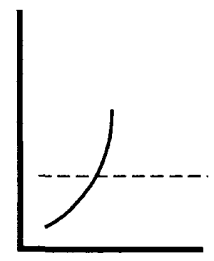

Intermediate Stage

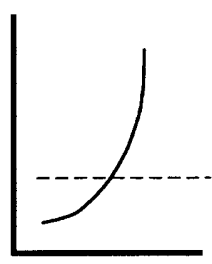

High Stage

Figure 3. Effect of changing stream stage on location of acoustic path in relation to vertical velocity profile.
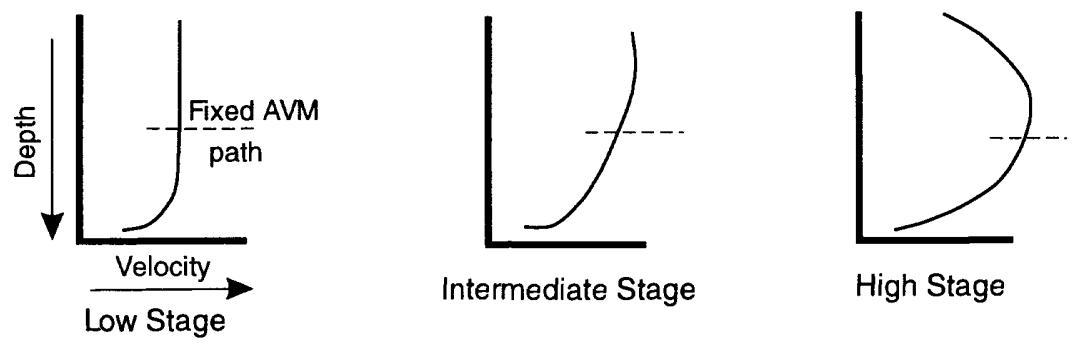

Figure 4. Effect of vertical velocity shapes on the relation of acoustic line velocity to mean channel velocity. 
Water surface

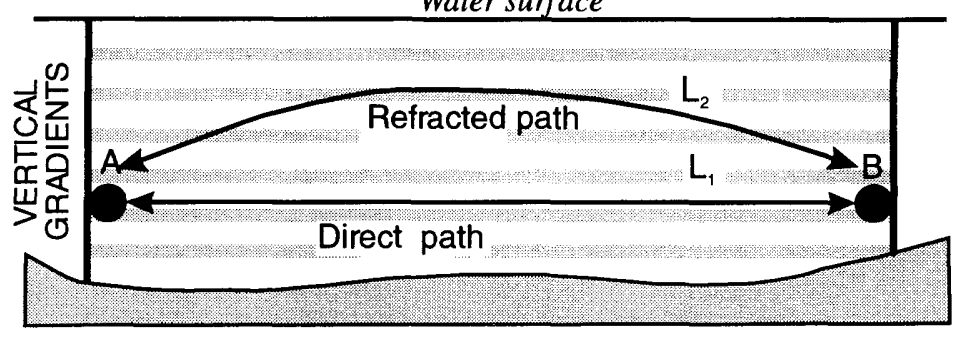

$L_{1}=$ DIRECT PATH LENGTH

$\mathrm{L}_{2}=$ PATH LENGTH WITH "RAY BENDING"

Channel bank

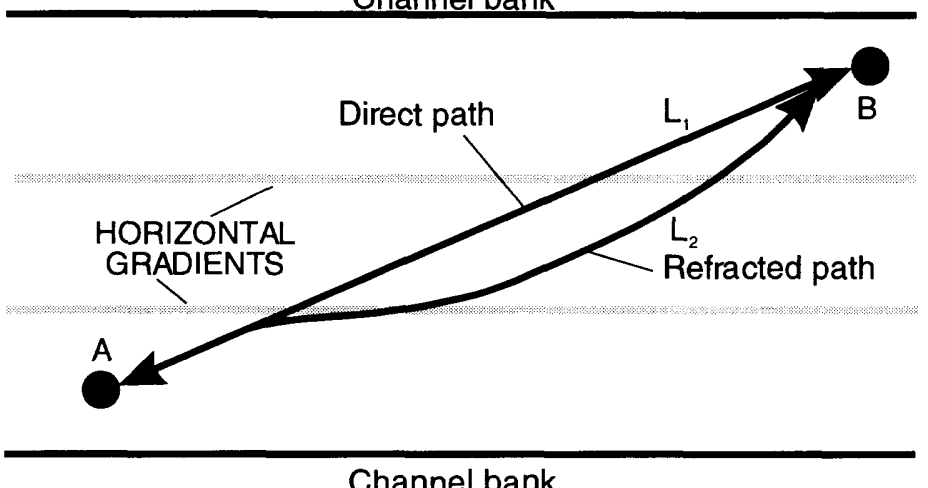

Channel bank

Figure 5. Vertical and horizontal ray-bending effects on acoustic path length. 
If ray bending is not so severe as to cause complete loss of signal, corrections to ray bending may be possible (Laenen, 1985). When ray bending occurs, the apparent speed of sound of the AVM signal (based on the average of upstream and downstream traveltimes) will be slower than when no ray bending occurs because of the longer refracted signal path length.

Unstable Flow Patterns-Unstable flow patterns can be the cause of varying flow angles or vertical velocity profiles in relation to the acoustic path angle and vertical location. Momentum driven flows (tidal) can present different flow patterns for the same velocity and stage, depending on the phase of the tide cycle. Control structures might be another reason for changing flow patterns, causing unstable flow angles and/or unstable vertical velocity profiles. Unstable flow patterns can greatly affect the accuracy of the calibration of AVM systems because they have a direct effect on the relation of acoustic line velocity to mean channel velocity. Figure 6 shows one possible scenario of unstable flow patterns due to control structures where the flow angle is variable depending on which part of the structure is the source of flow.

Tidal flows can sometimes occur in both upstream and downstream directions simultaneously during a tide cycle (fig. 7). This flow condition not only can affect the vertical distribution of upstream-downstream velocities, but can also distort cross-channel horizontal velocity distributions. During these conditions, the AVM might record velocities in one direction while the net flow is actually in the opposite direction. Therefore, velocity measurements need to be made to define the acoustic line velocity to mean channel velocity relation when these conditions occur.

Although AVM systems should be installed at sites that present the least number of factors affecting acoustic line velocity calibration, ideal conditions are seldom attainable. However, there are ways to account for some unstable flow patterns. For sites with variable horizontal flow angles, "cross-path" configurations (fig. 8A) can be used to account for the effect of changing flow angles. The error in acoustic line velocity of either path caused by a change in flow angle is offset by a compensating error in the other path. Therefore, the average of the two-line velocity measurements is far less susceptible to error than a single path. If a better representation of the vertical distribution of velocities is required on a continuous basis, a multiple vertical path configuration can be used (fig. 8B).
Vegetation-Excessive vegetation can affect AVM operation through signal absorption by the biomass or refraction of the acoustic signal by small gas bubbles entrapped in aquatic plants. Excessive vegetation can also result in changes of the effective flow area, the flow orientation with respect to the acoustic path, and the vertical velocity profile. How excessive vegetation can influence the effective flow area and the flow patterns through the AVM section is shown in figure 9. Vegetation can effectively change the horizontal angles of flow across the AVM measuring section and can also decrease the flow area, resulting in shifts or complete changes in the calibration ratings.

\section{COMPUTATION OF MEAN CHANNEL VELOCITY}

This section documents a technique that relates acoustic line velocity to mean channel velocity using a regression model calibrated with field measurements. As flow conditions change, it is impractical and unnecessary to account for all the physical factors which affect the acoustic line velocity to mean channel velocity relation (previously discussed). For example, an increase in stage would result in a change of several factors that affects the relation of acoustic line velocity to mean channel velocity; specifically, the position of the acoustic path in the vertical velocity profile, the shape of the velocity profile, the percentage of ungaged flow outside of the acoustic path, and other factors. However, it is only necessary to determine the change in the relation of acoustic line velocity to mean channel velocity, if the mean velocity will change as a result of changes in measurable variables, such as stage and line velocity. A series of discharge measurements made over the wide range of expected flow conditions is needed to define any change in the acoustic line velocity to mean channel velocity relation.

The model presented can be used to describe the mean channel velocity for a number of hydraulic conditions and can include several coefficients to account for all identifiable variables. The technique used to describe the relation is a least squares regression analysis in the single or multiple variable form, depending on the number of identified variables.

The model has the following equation form:

$$
\bar{V}=V_{l}\left(X_{i}+H X_{i+1}+\ldots+P_{n} X_{n}\right)+C
$$




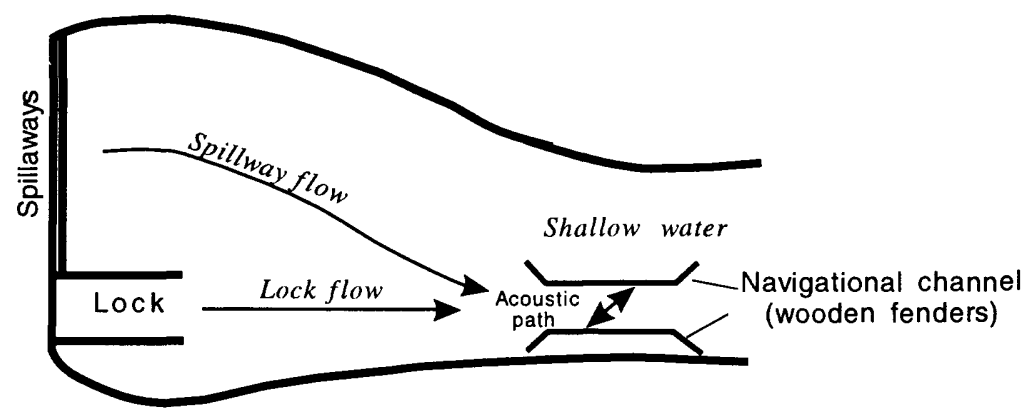

Figure 6. Variable flow angles through an acoustic velocity meter gaging site.

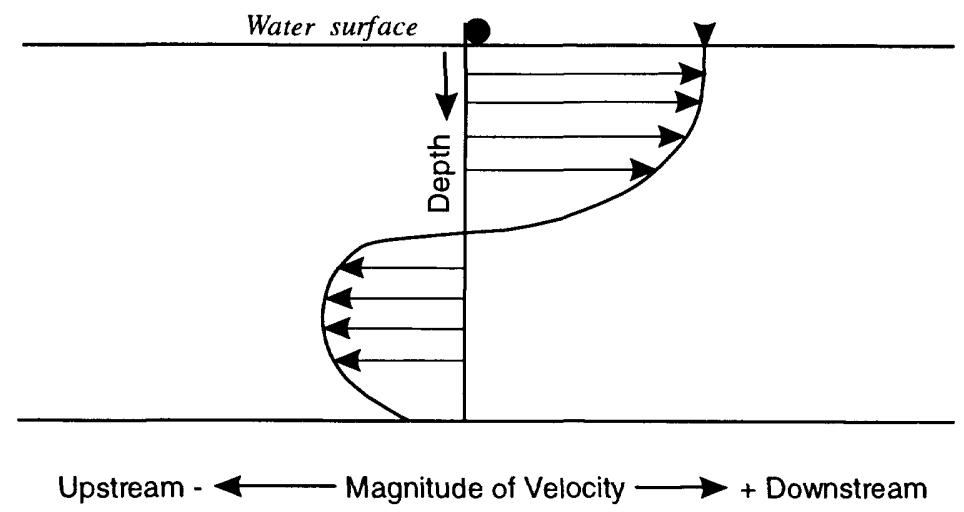

Figure 7. Distorted vertical velocity profile due to tidal effect. 


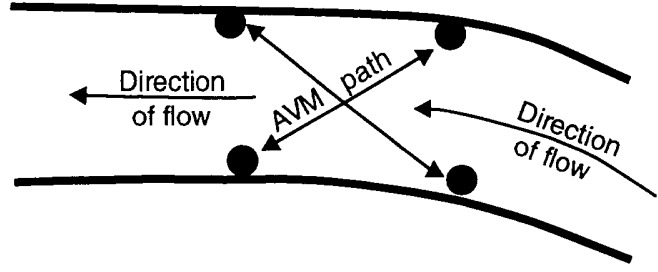

B

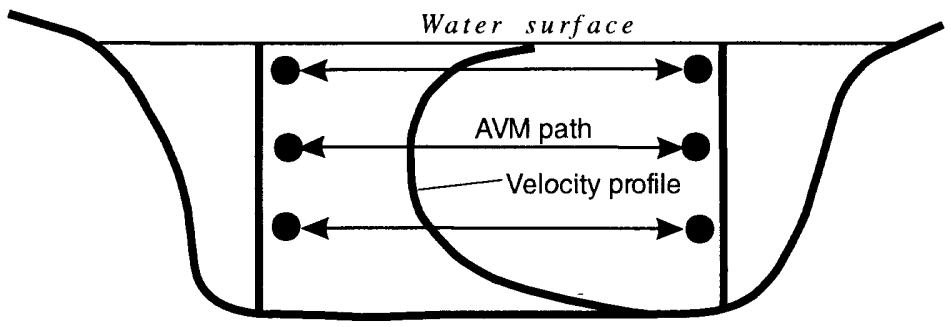

Figure 8. Multipath applications showing (A) cross-path setup and the horizontal flow angle problem and (B) multiple vertical paths and the velocity profile problem.

A

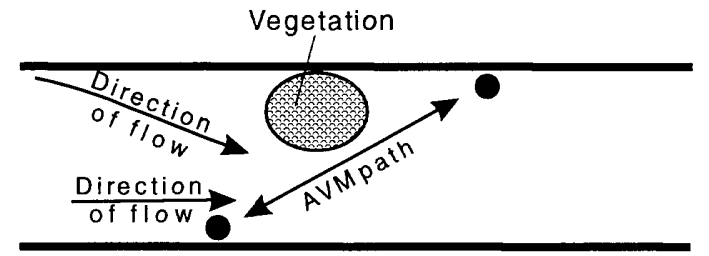

B

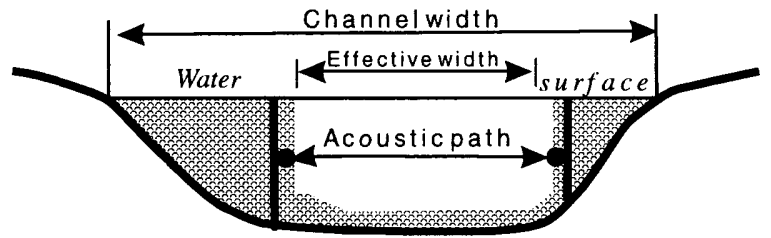

Figure 9. Plan view showing (A) flow angle caused by vegetation at acoustic velocity meter section and cross-section view showing (B) effective flow area significantly reduced by vegetation at acoustic velocity meter section. 
where $\bar{V}$ is mean channel velocity, $V_{l}$ is acoustic line velocity, $X_{i}$ through $X_{n}$ represent regression coefficients, $H$ is stage, $P_{n}$ represents other significant variables, and $C$ is the intercept or constant. This model allows for the fact that the expected change in $\bar{V}$ for a unit change in $V_{l}$ can be dependent on other secondary variables. These secondary variables, $P_{n}$, can be any variable, powers of these variables, cross products of two variables, or a function of several variables.

\section{Example 1}

This section presents a least squares regression analysis in the single variable form to relate acoustic line velocity to mean channel velocity. Based on previous field experience, acoustic line velocity is the only significant variable for many AVM sites in southern Florida. Effects from other variables are negligible. The resultant calibration equation then becomes:

$$
\bar{V}=V_{l} X_{i}+C
$$

To solve (5), a regression analysis is performed using $V_{l}$ as the independent variable and the measured (mean) channel velocity, $\bar{V}$, as the dependent variable. Careful consideration should be given to the intercept, $C$, because, depending on the hydraulic setting, it may or may not be negligible. For instance, tidal flow conditions (or flow reversal) may present nonzero mean channel velocities with a corresponding zero acoustic line velocity, and therefore, the intercept cannot be assumed negligible. Instrument offset (or bias) is also a factor that can be accounted for by $C$.

Example 1 represents a constant $\bar{V}$ to $V_{l}$ relation for all flow conditions and restricts the model to situations where the mean channel velocity is dependent only on the acoustic line velocity. The canal site selected for the single regression analysis is located in southern Florida (site A) and exhibits flow conditions that fit this form of the model. Graphical representation of the relation can be obtained by plotting acoustic line velocity against mean channel velocity with $X_{i}$ as the slope of the line and $C$ as the intercept (fig. 10). For this site, $X_{i}$ was 0.89 and $C$ was 0.003 . Table 1 presents the data used for calibration of the AVM system at site A and presents the results of the regression analysis.

\section{Example 2}

This section presents a least squares regression analysis in the multiple variable form to relate acoustic line velocity to mean channel velocity. Stage may be the second most significant variable commonly used in the model and when included in the equation, it adopts the following form:

$$
\bar{V}=V_{l}\left(X_{1}+H X_{2}\right)+C
$$

or:

$$
\bar{V}=V_{l} X_{1}+V_{l} H X_{2}+C
$$

where $H$ is stage. To solve (7), a multiple regression analysis is performed using $V_{l}$ and $V_{l} H$ as the independent variables and the measured (mean) velocity as the dependent variable. The intercept $C$ is given the same consideration as previously described.

Example 2 represents a relation between $\bar{V}$ and $V_{l}$ in which every expected change in $\bar{V}$ for a unit change in $V_{l}$ depends also on the stage (fig. 11). The canal site selected for the multiple regression analysis is located in southern Florida (site B) and exhibits the hydraulic characteristics that fit this form of the model. The intercept $C$ for this site was 0.003 (same as for site A). Because $\mathrm{C}$ was considered negligible for example 2 , the regression analysis was performed again, however, only this time forcing the model through the origin. Because the constant $C$ is considered to be zero for this example, (7) can take the form of:

$$
\bar{V} / V_{l}=X_{1}+H X_{2}
$$

Using (8), the velocity ratio can be plotted against the stage, $H$, with $X_{1}$ representing a constant (intercept) and $X_{2}$ representing the slope of the line. The dependency of the velocity relation $\left(V_{m} / V_{l}\right)$ to stage at site B is shown in figure 12 . Table 2 presents the data used for calibration of the AVM system at site $\mathrm{B}$ and presents the results of the regression analysis.

\section{Error Related to Velocity Rating}

The error related to any velocity rating is dependent not only on the operation and accuracy of the AVM system, but also on the instrument used to make discharge measurements necessary for calibration. 


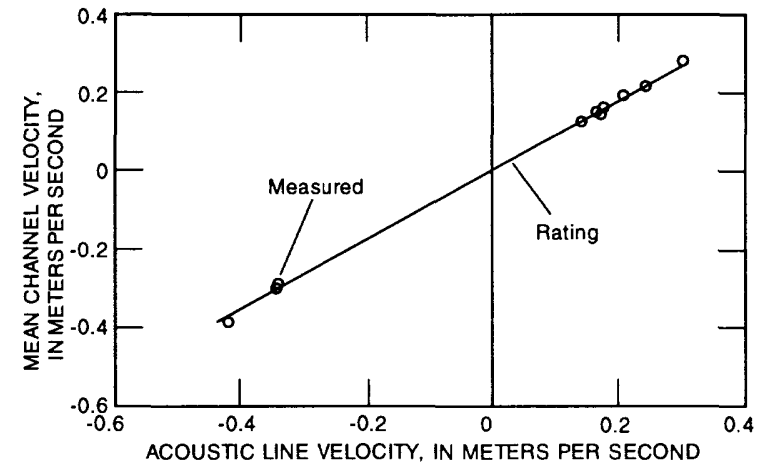

Figure 10. Relation of acoustic line velocity to mean channel velocity at site $A$.

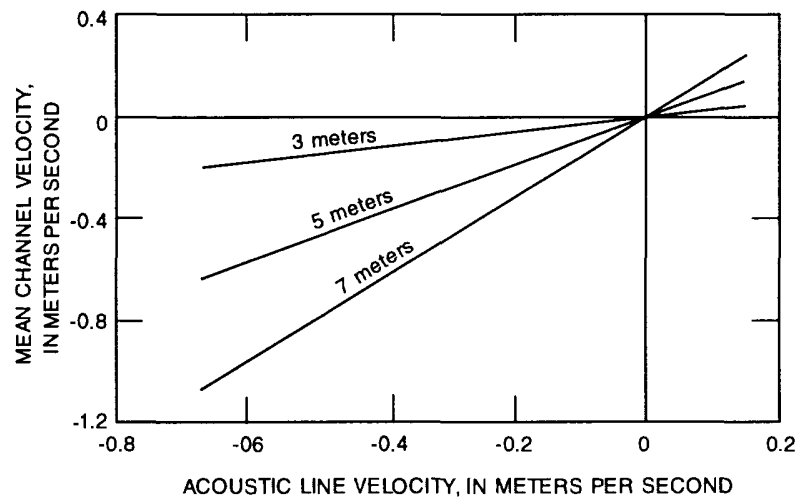

Figure 11. Relation of acoustic line velocity to mean channel velocity for three different stage levels at site B.

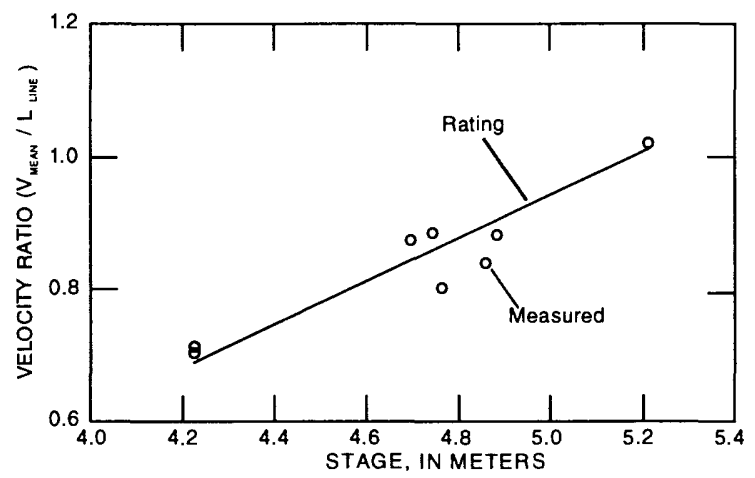

Figure 12. Relation of velocity ratio $\left(V_{n} / V_{n}\right)$ to stage at site $\mathrm{B}$. 
Table 1. Acoustic velocity meter system data and results of regression analysis at site $A$

[Measured velocity is measured discharge divided by cross sectional area. Index rated velocity is velocity calculated from regression equation. Rated discharge is index rated velocity multiplied by cross sectional area. Percent error of flow is (measured discharge - rated discharge)/measured discharge. Abbreviations: AVM, acoustic velocity meter; $\mathrm{m}^{3} / \mathrm{s}$, cubic meters per second; $\mathrm{m}$, meters; $\mathrm{m} / \mathrm{s}$, meters per second; and $\mathrm{m}^{2}$, square meters]

\begin{tabular}{rcccccccc}
\hline $\begin{array}{c}\text { Measured } \\
\text { discharge } \\
\left(\mathbf{m}^{3} / \mathbf{s}\right)\end{array}$ & $\begin{array}{c}\text { Mean } \\
\text { gage } \\
\text { height } \\
(\mathbf{m})\end{array}$ & $\begin{array}{c}\text { AVM } \\
\text { velocity } \\
(\mathbf{m} / \mathbf{s})\end{array}$ & $\begin{array}{c}\text { Cross } \\
\text { sectional } \\
\text { area } \\
\left(\mathbf{m}^{2}\right)\end{array}$ & $\begin{array}{c}\text { Measured } \\
\text { velocity } \\
(\mathbf{m} / \mathbf{s})\end{array}$ & $\begin{array}{c}\text { Index } \\
\text { rated } \\
\text { velocity } \\
(\mathbf{m} / \mathbf{s})\end{array}$ & $\begin{array}{c}\text { Rated } \\
\text { discharge } \\
\left(\mathbf{m}^{\mathbf{3}} / \mathbf{s}\right)\end{array}$ & $\begin{array}{c}\text { Percent } \\
\text { error of } \\
\text { flow }\end{array}$ & $\begin{array}{c}\text { Velocity } \\
\text { residuals }\end{array}$ \\
\hline 8.95 & 3.28 & 0.14 & 72.46 & 0.123 & 0.124 & 8.98 & -0.34 & 0.001 \\
17.31 & 3.45 & .25 & 77.63 & .223 & .218 & 16.94 & 2.22 & .005 \\
15.38 & 3.45 & .21 & 77.63 & .198 & .189 & 14.64 & 5.10 & .009 \\
11.76 & 3.37 & .18 & 75.26 & .156 & .156 & 11.76 & .02 & .000 \\
12.42 & 3.64 & .16 & 83.75 & .148 & .145 & 12.18 & 1.93 & .003 \\
12.35 & 3.61 & .18 & 82.95 & .149 & .156 & 12.96 & -4.71 & .007 \\
23.97 & 3.73 & .30 & 86.91 & .276 & .269 & 23.41 & 2.38 & .007 \\
-34.63 & 3.84 & -.42 & 90.63 & -.382 & -.369 & -33.44 & 3.55 & .013 \\
-32.65 & 3.67 & -.44 & 84.87 & -.385 & -.389 & -33.03 & -1.16 & .004 \\
-24.18 & 3.66 & -.34 & 84.56 & -.286 & -.302 & -25.56 & -5.21 & .016 \\
-25.99 & 3.75 & -.34 & 87.42 & -3.00 & -.304 & -26.61 & -2.31 & .004 \\
\hline
\end{tabular}

Table 2. Acoustic velocity meter system data and results of regression analysis at site $B$

[Measured velocity is measured discharge divided by cross sectional area. Index rated velocity is velocity calculated from regression equation. Rated discharge is index rated velocity multiplied by cross sectional area. Percent error of flow is (measured discharge - rated discharge)/measured discharge. Abbreviations: H, gate height; AVM, acoustic velocity meter; $\mathrm{m}^{3} / \mathrm{s}$, cubic meters per second; $\mathrm{m}$, meters; $\mathrm{m} / \mathrm{s}$, meters per second; and $\mathrm{m}^{2}$, square meters]

\begin{tabular}{|c|c|c|c|c|c|c|c|c|c|c|c|}
\hline \multirow{2}{*}{$\begin{array}{c}\text { Mea- } \\
\text { sured } \\
\text { dis- } \\
\text { charge } \\
\left(\mathrm{m}^{3} / \mathrm{s}\right)\end{array}$} & \multirow{2}{*}{$\begin{array}{c}\text { Mean } \\
\mathbf{H} \\
(\mathrm{m})\end{array}$} & \multirow{2}{*}{$\begin{array}{c}\text { Mean } \\
\text { AVM } \\
\text { veloc- } \\
\text { ity } \\
(\mathrm{m} / \mathrm{s})\end{array}$} & \multirow{2}{*}{$H \times V_{1}$} & \multirow{2}{*}{$\begin{array}{c}\text { Cross } \\
\text { sec- } \\
\text { tional } \\
\text { area } \\
\left(m^{2}\right)\end{array}$} & \multirow{2}{*}{$\begin{array}{l}\text { Mea- } \\
\text { sured } \\
\text { veloc- } \\
\text { ity } \\
(\mathrm{m} / \mathrm{s})\end{array}$} & \multicolumn{2}{|c|}{$\begin{array}{l}\text { Velocity } \\
\text { relation } \\
\left(V_{m} N_{1}\right)\end{array}$} & \multirow{2}{*}{$\begin{array}{c}\text { Index } \\
\text { rated } \\
\text { veloc- } \\
\text { ity } \\
(\mathrm{m} / \mathrm{s})\end{array}$} & \multirow{2}{*}{$\begin{array}{l}\text { Rated } \\
\text { dis- } \\
\text { charge } \\
\left(\mathrm{m}^{3} / \mathrm{s}\right)\end{array}$} & \multirow{2}{*}{$\begin{array}{l}\text { Percent } \\
\text { error of } \\
\text { flow }\end{array}$} & \multirow{2}{*}{$\begin{array}{l}\text { Veloc- } \\
\text { ity } \\
\text { resid- } \\
\text { uals }\end{array}$} \\
\hline & & & & & & $\begin{array}{l}\text { Mea- } \\
\text { sured }\end{array}$ & $\begin{array}{l}\text { Esti- } \\
\text { mated }\end{array}$ & & & & \\
\hline 5.09 & 4.22 & 0.216 & 0.91 & 33.34 & 0.153 & 0.71 & 0.69 & 0.150 & 4.99 & 2.11 & 0.003 \\
\hline 4.94 & 4.22 & .207 & .88 & 33.34 & .148 & .71 & .69 & .143 & 4.78 & 3.42 & .005 \\
\hline 3.04 & 4.70 & .082 & .39 & 42.22 & .072 & .87 & .84 & .070 & 2.94 & 3.52 & .004 \\
\hline 3.05 & 4.76 & .088 & .42 & 43.04 & .071 & .80 & .86 & .076 & 3.29 & -7.19 & .005 \\
\hline-9.32 & 4.74 & -.244 & -1.16 & 43.11 & -.216 & .89 & .86 & -.210 & -9.04 & 3.11 & .006 \\
\hline-10.02 & 4.86 & -.262 & -1.27 & 45.43 & -.221 & .84 & .90 & -.235 & -10.68 & -6.18 & .014 \\
\hline-14.84 & 4.88 & -.366 & -1.79 & 45.93 & -.323 & .88 & .91 & -.331 & -15.21 & -2.41 & .008 \\
\hline-35.88 & 5.21 & -.664 & -3.46 & 52.90 & -.678 & 1.02 & 1.01 & -.673 & -35.58 & .83 & .005 \\
\hline
\end{tabular}


This rating error can be reported in different ways, including percent deviation from measured velocity, standard error of estimate $(\bar{V})$ for the regression analysis, or residuals (measured velocity - rated velocity [or discharges]). The type of error reported depends on the method of reporting flow. For example, tidal flows can be reported as "net flow" per cycle, in cubic meters per second and the error also reported in cubic meters per second. Importantly, if the estimates of $\bar{V}$ are to be used in discharge calculations, the total error involved in the process will also include errors in the measurement of channel area.

\section{CONCLUSIONS}

AVM systems have proven to be accurate and reliable instruments for measuring the velocity of water in open channels by means of an acoustic signal that travels faster downstream than upstream. The accuracy of the measurements depends on the calibration process that relates acoustic line velocity to mean channel velocity. AVM systems provide a line velocity measurement at one or several points in the vertical. The acoustic line velocity is an integrated velocity measurement along a line or path between the acoustic transducers.

AVM systems, often viewed as discharge meters, are actually velocity measuring devices. Various physical factors (channel characteristics and flow conditions) can affect the relation of acoustic line velocity to mean channel velocity, and in turn, affect the determination of discharge. The ideal AVM gaging site minimizes most of these factors. The fewer complicating factors involved, the simpler, more reliable, and precise the rating becomes.

Analyses were performed at two canal sites in southern Florida to relate acoustic line velocity to mean channel velocity by means of a regression model calibrated with field measurements. Discharge measurements were made over a range of flow conditions to define the relation between acoustic line velocity to mean channel velocity at each site. Example 1 produced a simple regression model with acoustic line velocity as the only variable, and example 2 produced a more complex model that included both acoustic line velocity and water-surface elevation as variables. Other field situations may require that different variables be included in the regression model. Results were used to demonstrate how single and multiple linear regression models can be used to calibrate acoustic line velocity to mean channel velocity and discharge.

\section{REFERENCES CITED}

Laenen, Antonius, 1985, Acoustic velocity meter systems: U.S. Geological Survey Techniques of WaterResources Investigations, book 3, chap. A17.

Laenen, Antonius, and Curtis, R.E., 1989, Accuracy of acoustic velocity metering systems for measurement of low velocity in open channels: U.S. Geological Survey Water-Resources Investigations Report 89-4090, 15 p.

Laenen, Antonius, and Smith, Winchell, 1983, Acoustic systems for the measurement of streamflow: U.S. Geological Survey Water-Supply Paper 2213.

Patino, Eduardo, 1996, Feasibility of using acoustic velocity meters for estimating highly organic suspended-solids concentrations in streams: U.S. Geological Survey Open-File Report 96-137, 28 p. 